\title{
Experimental Gas-Phase Spectroscopy of Carbon Molecular Structures and Diffuse Interstellar Bands
}

\author{
J. JANČA ${ }^{1}$, M. PLONKA ${ }^{2}$, \\ M. ŠOLC ${ }^{3}$ and M. VETEŠNÍK ${ }^{2}$
}

${ }^{1}$ Plasma-Chemistry Laboratory, Masaryk University, Brno, Czech Republic

${ }^{2}$ Department of Theoretical Physics and Astrophysics

Masaryk University, Brno, Czech Republic

${ }^{3}$ Astronomical Institute of Charles University, Prague, Czech Republic

Preliminary results of an experimental study of ultraviolet to near-infrared gas-phase spectra of possible candidate materials for Diffuse Interstellar Bands (DIBs) are presented. The experimental technique enabling the study of potential carriers of DIBs in conditions that mimic the natural environment of carbon-star atmospheres is described. Conclusions concerning some carriers to be searched for (including molecular features at $\lambda 2150, \lambda 2600, \lambda 5844, \lambda 5850$, $\lambda 9577$, and $\lambda$ 9632) are also discussed.

We have searched for the spectral features of fullerene $\mathrm{C}_{60}$ in the ultraviolet at wavelengths corresponding to the measurements of Leach et al. (1992, Chem. Phys., 160, 451). Following a detailed analysis, no fullerene spectral features have been detected, although they could be fully masked by molecular absorptions that cannot be reduced. Such a conclusion holds true also for the observed feature at $\lambda 2150$ which can be identified with the rovibrational spectra of various types of molecules like $\mathrm{C}_{2} \mathrm{~N}_{2}, \mathrm{CO}, \mathrm{CO}^{+}$, and $\mathrm{N}_{2}$, while the true fullerene profile should be much broader.

Our attempt to throw light upon the DIBs at $\lambda 5844$ and $\lambda 5850$, recently studied by Jenniskens et al. (1996, $A \& A, 313,649)$, has resulted in the conclusion that two spectral features observed in a helium atmosphere enriched with hydrogen atoms could be, under suitable conditions, considered as counterparts of the DIBs. This statement is supported by the structure seen in the feature at $\lambda 5844$.

We have also considered two new DIBs at $\lambda 9577$ and $\lambda 9632$, theoretically attributed to interstellar $\mathrm{C}_{60}^{+}$(Foing \& Ehrenfreund 1995, in The Diffuse Interstellar Bands, ed. Tielens and Snow, p. 65). Unfortunately, as both of these DIBs coincide with the $(2,1)$ band of the red system $\left({ }^{2} \Pi-{ }^{2} \Sigma\right)$ of $\mathrm{CN}$, it is hard to say that the spectral features observed by us are due to $\mathrm{C}_{60}^{+}$. 